\title{
The major hormone-specific discontinuous epitopes on human chorionic gonadotrophin
}

\section{Charrel-Dennis*, A M Jackson*, T Lund, A J Lapthorn'1, P Berger², I M Roitt and P J Delves}

\author{
Department of Immunology and Molecular Pathology, University College London, London, UK \\ ${ }^{1}$ Department of Chemistry, Joseph Black Building, University of Glasgow, Glasgow, UK \\ ${ }^{2}$ Institute for Biomedical Ageing Research, Austrian Academy of Sciences, Innsbruck, Austria \\ (Requests for offprints should be addressed to I M Roitt, Department of Immunology and Molecular Pathology, University College London, \\ 46 Cleveland Street, London W1T 4JF, UK; Email: i.roitt@ucl.ac.uk) \\ *(M Charrel-Dennis and A M Jackson contributed equally to the work)
}

M Charrel-Dennis is currently at Channing Laboratory, Harvard Medical School, Boston, Massachusetts, USA

A M Jackson is currently at Discerna Ltd, Babraham Research Campus, Cambridge, UK

\begin{abstract}
The hormone human chorionic gonadotrophin (hCG) shows extensive sequence homology with $\mathrm{LH}$. Thus, many of the antigenic epitopes on hCG are shared with LH, leading to immunological cross-reaction between these two hormones. Anti-fertility and anti-cancer vaccines based upon hCG should ideally target only the hCG-specific epitopes. The hCG-unique linear epitopes located in the C-terminal peptide of the hCG $\beta$-chain are well characterised. In contrast, the hCG-specific discontinuous epitopes, termed $\beta 1, \beta 6$ and $\beta 7$, have remained poorly defined. By generating hCG $\beta$-chain molecules containing single amino acid substitutions we have identified R10, N13, R60 and Q89 as being important in the formation of the $\beta 1$ epitope, with R60 providing a particularly dominant residue. We also show that the amino acid residue Q89 contributes to the $\beta 7$ epitope, whilst D61 plays a role in both the $\beta 6$ and $\beta 7$ epitopes.
\end{abstract}

Journal of Molecular Endocrinology (2004) 32, 571-581

\section{Introduction}

The glycoprotein hormone family is composed of human chorionic gonadotrophin (hCG), luteinising hormone $(\mathrm{LH})$, follicle-stimulating hormone and thyroid-stimulating hormone. These hormones are heterodimers comprising a common $\alpha$-chain and a hormone-specific $\beta$-chain, both of which are glycosylated with N-linked carbohydrate (the $\beta$-chain of hCG also bearing O-linked carbohydrate). Both subunits share a common fold of two beta hairpins and a variable loop region and are rich in disulphide bonds, three of which form the central structural motif called a cysteine knot (Lapthorn et al. 1994, Wu et al. 1994). The two subunits are symmetrically interlocking, with the $\beta$-chain embracing its $\alpha$-chain counterpart with a loop structure that is referred to as the 'seat-belt' (Lapthorn et al. 1994).
hCG is a pregnancy-related hormone produced by the pre-implantation blastocyst and subsequently by the trophoblast (Fox \& Kharkongor 1970). Because hCG is implicated in implantation in the uterus and is essential for promoting the secretion of oestrogen and progesterone from the corpus luteum, and thus for maintaining the endometrium in a receptive state, blocking the action of this hormone with antibodies prevents pregnancy. Thus, vaccines based on this hormone have been developed as anti-fertility agents (Stevens 1999, Talwar 1999, Delves et al. 2002) and one such vaccine has successfully completed phase II efficacy trials (Talwar et al. 1994). Furthermore, hCG-based vaccines may have utility for the treatment of certain hormone-dependent cancers (Triozzi \& Stevens 1999, Moulton et al. 2002). However, there is an $85 \%$ sequence homology between the first 112 amino acids of the $\beta$-chains of 
hCG and LH, leading to immunological crossreaction between the two hormones due to a number of shared epitopes. An anti-hCG response which cross-reacts with LH may be undesirable as $\mathrm{LH}$ is not a pregnancy-specific hormone but a critical hormone in the process of ovulation and production of female sex hormones. Whilst adverse effects of antibody responses to LH have not been observed (Talwar 1999), it is possible that long-term exposure to a high level of LH-cross-reactive antibodies might lead to undesirable side-effects in hCG-vaccinated individuals (Dirnhofer et al. 1994).

A number of epitopes on hGG have been defined with monoclonal antibodies (mAbs) (Berger et al. 2002). Two linear epitopes, $\beta 8$ and $\beta 9$, have been mapped within the amino acid sequence 109-145, which constitutes the G-terminal peptide (CTP) of the molecule, a structure which is unique to hCG and is believed to adopt an extended, poorly ordered conformation. All the other B-cell epitopes on the hCG $\beta$-chain are discontinuous and therefore require the correct folding of the hormone. The $\beta 1$ epitope is of particular interest as, like the CTP, it is hCG-specific and thus also a desirable epitope to incorporate into an hCG-based vaccine. Structural assignment of the $\beta 1$ epitope region on the hCG $\beta$-chain has remained elusive. The majority of surface differences between hCG and $\mathrm{LH}$ on the body of the $\beta$-chain are located in a defined cluster close to the central cysteine knot (Fig. 1). We therefore hypothesised that this region must constitute the $\beta 1$ epitope, and residues within this area were selected for mutation. Residues R8, R10 and Q89 are unique to hCG and are present in a region protruding from the body of the molecule. In the three-dimensional structure, hCG-specific residues Q89 and R10 are in close proximity to R60 and D61. Thus, residues R8, R10, R60, D61 and Q89 were selected for mutation and the effects of these mutations on the establishment of contact with the antibody paratope analysed. LH and hCG differ in their carbohydrate content (Kessler et al. 1979), the hCG $\beta$-chain bearing two $\mathrm{N}$-linked carbohydrate structures at N13 and N30, whereas the LH $\beta$-chain lacks the glycosylation on N13 due to the replacement of threonine at position 15 with a isoleucine in the LH sequence, abolishing the consensus sequence for $\mathrm{N}$-linked glycosylation. Given that the $\beta 1$ epitope is present only on the
hCG molecule, it is possible that the presence of the carbohydrate structure might induce minor conformational changes in the structure of hCG and thereby indirectly contribute to the generation of this epitope. Thus, residue N13 was also targeted for mutation. We additionally investigated amino acids contributing to the $\beta 6$ and $\beta 7$ epitopes associated with two discrete antigenic regions accessible only on the free $\beta$-chain, and therefore presumed to involve residues located at the $\alpha / \beta$ interface (Berger et al. 2002).

\section{Material and methods}

\section{mAbs}

All mAbs have been described previously (Berger et al. 1990, Jackson et al. 1996) and recognise various epitopes as indicated; INN-2 mAb $(\beta 1)$, INN-32 mAb $(\beta 1)$, INN-111 mAb $(\beta 3)$, INN-24 mAb ( $\beta 4)$, INN-58 mAb ( $\beta 5)$, INN-51 mAb ( $\beta 3 / 5)$, INN-20 mAb $(\beta 3 / 5)$, INN-64 mAb ( $\beta 6)$, INN-68 $\mathrm{mAb}(\beta 7)$ and 3E2 mAb ( $\beta 3 / 5)$. The OT3A2 mAb recognises the linear $\beta 8$ CTP epitope 133-139. Isotype control mAbs for flow cytometry were QB-END/10 anti-CD34 (Quantum Biosystems, Cambridge, UK) and TT04 anti-tetanus toxoid (National Institute for Biological Standards and Control, Potters Bar, Herts, UK) (Sheppard et al. 1984).

\section{Construction of the mutants}

The generation of the expression vector construct has been previously described (Jackson et al. 1996). The mutants were produced by an overlap PGR mutagenesis method (Horton \& Pease 1991) using hCG $\beta$-chain cDNA as a template. The primer sequences are listed in Table 1 . The mutated genes were initially cloned into pBluescript (Stratgene, Ceder Creek, TX, USA) and the position of the desired mutations, and the absence of any undesired mutations, confirmed by sequencing. The wild-type and mutants were then subcloned into a pCDM8 construct (Seed 1987), in which a DNA fragment coding for the first 17 membraneproximal residues, the transmembrane region and the cytoplasmic tail of the $\mathrm{H} 2-\mathrm{D}^{\mathrm{b}}$ protein had been previously cloned in frame with the hCG $\beta$-chain gene (Jackson et al. 1996). The fragments containing the wild-type or the mutants R10E, 


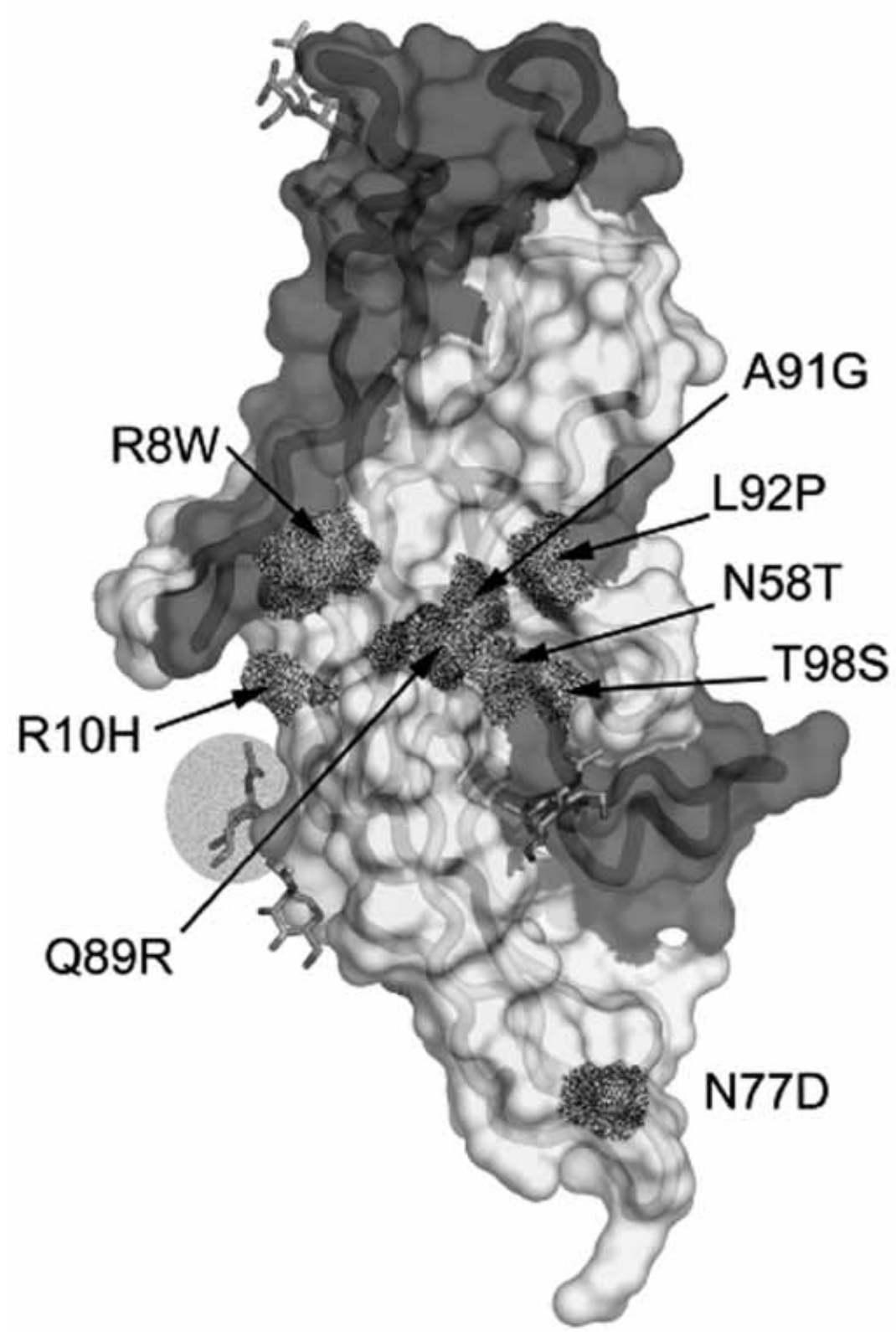

Figure 1 Surface exposed differences between hCG and LH. The hCG heterodimer structure (PDB 1HRP, Lapthorn et al. 1994) in surface representation with the position of individual amino acid residues which are changed in LH highlighted. The $\alpha$-chain surface is shaded in dark grey and the $\beta$-chain surface in light grey. The $\mathrm{N}$-acetylglucosamine sugars visible in the hCG crystal structure which are attached at N13 and N30 of the $\beta$-chain and N52 and N78 of the $\alpha$-chain are shown in stick representation. These provide an indication of where the biantennary $\mathrm{N}$-linked oligosaccharides cover the structure. The sugar at $\mathrm{N} 13$ is highlighted as this is absent in LH due to the change T15I. The $\beta$-chain CTP is not resolved in the X-ray structure and is therefore not shown. 
Table 1 Primer sequences used for construction of the hCG $\beta$-chain mutants

\section{Primer sequences}

\begin{tabular}{|c|c|c|}
\hline \\
\hline \multirow{2}{*}{ R8L } & Sense: & 5' GGCCACTGTGCCGCCCCATCAATGCCACC 3' \\
\hline & Antisense: & 5' GGGCGGCACAGTGGCCGAAGCGGCTCC 3' \\
\hline \multirow[t]{2}{*}{ R10E } & Sense: & 5' GCGAGCCCATCAATGCCACCCTGGCTGTGG 3' \\
\hline & Antisense: & 5' GGCATTGATGGGCTCGCACCGTGGCCGAAGC 3' \\
\hline \multirow[t]{2}{*}{ N13E } & Sense: & 5' GCCACCCTGGCTGTGGAG 3' \\
\hline & Antisense: & 5' CTCCACAGCCAGGGTGGCCTCGATGGGGCGCACCGTGG 3' \\
\hline $\mathrm{R} 60 \mathrm{H}+\mathrm{R} 63 \mathrm{~S}+\mathrm{E} 65 \mathrm{~A}$ & \multicolumn{2}{|c|}{$\begin{array}{l}\text { An additional mutation (R60H) generated by a PCR error during an initial attempt at constructing } \\
\text { R63S+E65A (see R63S+E65A below for primer sequences) }\end{array}$} \\
\hline \multirow[t]{2}{*}{ D61R } & Sense: & 5' GTGCGCTTCGAGTCCATCCGG 3' \\
\hline & Antisense: & 5' GATGGACTCGAAGCGCACCKSGCGGTAGTT 3' \\
\hline \multirow[t]{2}{*}{ R63S+E65A } & Sense: & 5' GTGTCCTTCGCCTCCATCCGGCTCCCTGGC 3' \\
\hline & Antisense: & 5' GGAGGCGAAGGACACATCGCGGTAGTTG 3' \\
\hline \multirow[t]{2}{*}{ Q89R } & Sense: & 5' CAGCTGTCGCTGTGCACTCTGCCGCCGC 3' \\
\hline & Antisense: & 5' GCACAGCGACAGCTGAGAGCCACGGC 3' \\
\hline \multirow[t]{2}{*}{ A91N } & Sense: & 5' GTCAATGTAACCTCTGCCGCCGCAGCACC 3' \\
\hline & Antisense: & 5' GCAGAGGTTACATTGACAGCTGAGAGCC 3' \\
\hline \multirow[t]{2}{*}{ L92P } & Sense: & 5' AATGTGCACCCTGCCGCCGCAGCACCACA 3' \\
\hline & Antisense: & 5' CGGCAGGGTGCACATTGACAGCTGAGAGC 3' \\
\hline
\end{tabular}

N13E and D61R hCG $\beta$-chain-MHC fusion genes were further subcloned into pcDNA3 as NotI-SalI fragments.

\section{Expression of wild-type and mutated hCG $\beta$-chains}

Dulbecco's modified Eagle's medium (DMEM), fetal calf serum (FCS), Hank's balanced salt solution (HBSS), trypsin-versene solution, chloroquinine, gentamycin and G-418 were purchased from Invitrogen-Life Technologies Ltd (Paisley, Renfrewshire, UK). DMEM was supplemented with $4 \mathrm{mM} \mathrm{L}$-glutamine, $1.5 \mathrm{~g} / 1$ sodium bicarbonate, $4.5 \mathrm{~g} / 1$ glucose and $1 \mathrm{mM}$ sodium pyruvate. Chinese hamster ovary $(\mathrm{CHO})$ cells were cultured in DMEM containing $10 \%$ FCS and $1 \%$ penicillin-streptomycin at $37^{\circ} \mathrm{C}$ in a $5 \% \quad \mathrm{CO}_{2}$ incubator in a humidified atmosphere. Liposomemediated transfection of the $\mathrm{CHO}$ cells was undertaken using cationic liposomes, formulated from 3-beta $\left[\mathrm{N}-\left(\mathrm{N}^{\prime}, \mathrm{N}^{\prime}\right.\right.$-dimethylaminoethane) carbamoyl]-cholesterol and dioleoyl L-alphaphosphatidylethanolamine, kindly provided by Dr Karl Murray (Department of Psychiatry and Behavioural Sciences, University College London, London, UK) (McQuillin et al. 1997). The cells were transfected at three different $(\mathrm{w} / \mathrm{w})$ ratios of plasmid DNA to liposomes $(1 / 1,1 / 3,1 / 6)$. The appropriate amount of DNA and liposomes were added to $400 \mu \mathrm{l}$ serum-free DMEM, and the mix vortexed and then incubated for $30 \mathrm{~min}$ at room temperature. This mix was deposited onto 50\% confluent cells present in a well of a Costar six-well plate (Corning BV, Schiphol-Rijk, The Netherlands). After $2 \mathrm{~h}$ of incubation, growth medium was added to each well and the cells harvested $48 \mathrm{~h}$ later. To generate stable cell lines expressing wild-type or mutants R10E, N13E and D61R of the hCG $\beta$-chain, or expressing either green fluorescent protein (GFP) or only neomycin resistance, the cells were allowed to grow for 5 days post-transfection under non-selective conditions prior to selection of antibiotic-resistant clones in growth medium supplemented with $800 \mu \mathrm{g} / \mathrm{ml}$ G-418 sulphate. After 14 days (2 days after $100 \%$ of the mock transfected cells had died under the G-418 selection) single colonies were aspirated and transferred to individual wells of a 24-well plate containing growth medium-G-418. Individual clones were expanded and analysed.

\section{Flow cytometry}

Cells $\left(2 \times 10^{5}\right)$ were stained with $1-10 \mu \mathrm{g} / \mathrm{ml}$ primary antibody in $2 \%(\mathrm{w} / \mathrm{v}) \mathrm{BSA}, 0 \cdot 02 \%(\mathrm{v} / \mathrm{v})$ sodium azide in HBSS and incubated on ice for 45 min. After three washes in HBSS, the cells 
were resuspended in fluorescein isothiocyanate (FITG)-conjugated $\mathrm{F}\left(\mathrm{ab}^{\prime}\right)_{2}$ rabbit anti-mouse IgG (Dako, High Wycombe, Bucks, UK) in the above buffer and incubated on ice for $30 \mathrm{~min}$. After three washes, the cells were resuspended in HBSS and analysed by fluorescence-activated cell sorting using a Becton-Dickinson Facscan (Becton Dickinson, Mountain View, CA, USA). The gate was set on the negative control, an irrelevant isotype-matched antibody. All mAbs were titrated to give roughly the same staining intensity on the wild-type surface-expressed hCG $\beta$-chain. Each mutant/ $\mathrm{mAb}$ combination was tested at least three times. The relative expression of each epitope normalised to that of CTP $\beta 8$ was calculated using the following ratio for each antibody: relative expression $=($ percent positive cells obtained with the antibody)/(percent positive cells obtained with OT3A2). Any reduction in binding $<20 \%$ was disregarded.

The ratios were statistically analysed. An F-test showed that the variance between each population was the same and therefore a Student's $t$-test was utilised to assess statistical significance.

\section{Results}

\section{Expression of wild-type and mutant hCG $\beta$-chain on $\mathrm{CHO}$ cells}

The cell-surface expression of the chimeric $\beta$-hCG proteins was quantified using the OT3A2 mAb, which recognises a CTP linear epitope on the hCG $\beta$-chain of each mutant molecule. Similarly to our previous studies using COS7 cells (Jackson et al. 1996), both wild-type and mutant hCG $\beta$-chains were capable of being expressed on the surface of CHO cells (Fig. 2). Furthermore, as assessed using ten different conformation-dependent mAbs, surface expression of recombinant wild-type hCG $\beta$-chain on the $\mathrm{CHO}$ cells also did not dramatically affect the folding of the molecule (Fig. 3a). The amount of antibody per cell required to obtain a similar level of staining for each epitope was therefore established using $\mathrm{CHO}$ cells expressing wild-type hCG $\beta$-chain.

\section{Mutations affecting the $\beta 6$ and $\beta 7$ epitopes}

The $\beta 3, \beta 4$, and $\beta 5$ epitopes were recognised equally well on the mutants R8L, A91N, L92P,
R63S+E65A (data not shown), R10E, N13E, D61R, Q89R and R60H+R63S+E65A (Fig. 3b-f) indicating that these mutations did not substantially modify the overall structure of the protein. However, the Q89R mutation led to an $80 \%$ decrease in the binding of the INN-68 $\mathrm{mAb}$ recognising the $\beta 7$ epitope $(P<0 \cdot 0005$, Fig. 3e). The glutamine at position 89 is most likely located within the immediate environment of the $\beta 7$ epitope. Modification of the aspartic acid at position 61 (D61R) decreased the binding of the $\beta 6$ - and $\beta 7$-specific antibodies by approximately $30 \%(P<0.005$ for INN-64 and $<0.05$ for INN-68) (Fig. 3d).

\section{Mutations affecting the $\beta 1$ epitope}

Mutating the hCG $\beta$ chain-specific residues R8 to a glutamic acid, A91 to an asparagine or L92 to proline as single point mutants did not affect the binding of any of the mAbs used including the hCG-specific $\beta 1$ epitope antibodies INN-2 and INN-32 (data not shown). However, recognition of the mutant R10E by the mAbs INN-2 and INN-32 was reduced by approximately $30 \% \quad(P<0 \cdot 005)$ compared with the wild-type clone (Fig. 3b), but none of the other epitopes were affected by this mutation. Substituting Q89 for arginine led to a decrease in binding on the $\beta 1$-specific antibodies of about $30 \%(P<0 \cdot 005$, Fig. 3e). The R60H+ $\mathrm{R} 63 \mathrm{~S}+\mathrm{E} 65 \mathrm{~A}$ triple mutation totally abolished the recognition of the $\beta 1$ epitope (Fig. 3f) $(P<0 \cdot 0005)$, but had no effect on any of the other epitopes. In contrast, the R63S+E65A double mutation bound all antibodies equally as well as the wild-type $\beta$-hCG (data not shown), thereby implying that the $\mathrm{R} 60 \mathrm{H}$ mutation is responsible for the abolition of the $\beta 1$ epitope. The likely approximate location of the $\beta 1$ epitope, indicating the position of the amino acids R10, R60 and Q89, whose mutation led to a reduction in binding of the $\beta 1$-specific mAbs, is shown in Fig. 4.

\section{Role of carbohydrate in antibody binding}

hCG is the most highly glycosylated member of the glycoprotein hormone family of proteins, with two N-linked biantennary complex carbohydrate chains per subunit and four O-linked carbohydrates in the unique $\beta$-subunit CTP. LH in contrast has only one $\beta$ chain carbohydrate due to the replacement 
(a) GFP

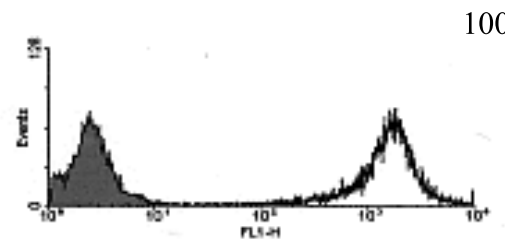

(b) Wild-type

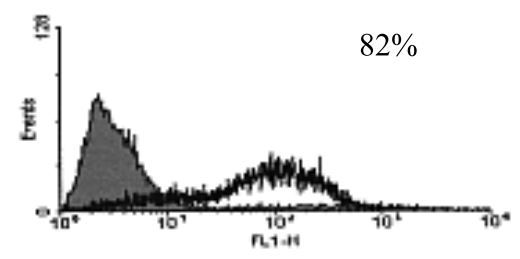

(d) $\quad$ N13E

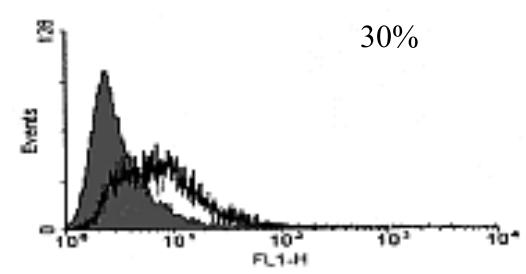

(c) $\mathrm{R} 10 \mathrm{E}$

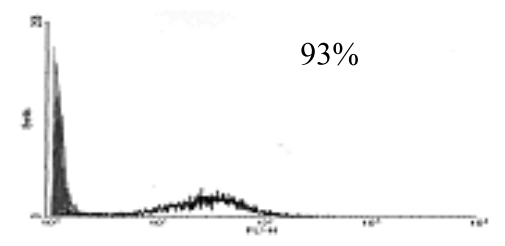

(e) D61R

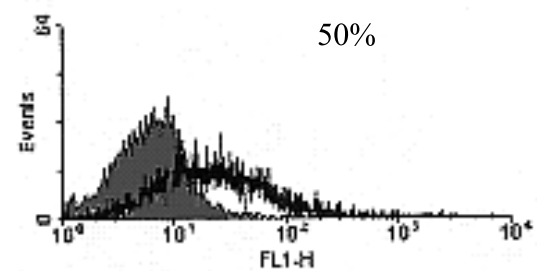

Figure 2 Stable expression of wild-type and mutated hCG $\beta$-chain on the cell surface of $\mathrm{CHO}$ cells. Events (5000) are shown for $\mathrm{CHO}$ cells optimally expressing GFP, wild-type hCG $\beta$-chain or, as representative examples, mutants R10E, N13E and D61R stained with OT3A2 mAb (black), which recognises the linear $\beta 8$ epitope (amino acids 133-139) of the hCG $\beta$-chain CTP. Expression levels often varied when the same plasmid was used on different occasions. The negative isotype-matched controls are shown in red. The indicated percentage of cells expressing the chimeric construct was determined by the cells with a higher fluorescence intensity than the negative isotype-matched control.

of threonine at position 15 with an isoleucine in the consensus sequence for N-linked glycosylation. These differences in glycosylation could significantly affect the regions of the $\beta$-subunit surface accessible to an antigenic response in the two hormones. As expected, the mutation N13E did not substantially affect the $\beta 3, \beta 4$ and $\beta 5$ epitopes as these are distant from the glycosylation site. However, the mutation led to an approximately $20 \%$ increased recognition by the $\beta 1$-specific antibody INN-32 compared with binding to the wild-type protein $(P<0 \cdot 05)$ (Fig. 3c). This suggests that the carbohydrate associated with residue 13 partly interferes with the binding of the antibody INN-32 and is therefore at the periphery of the $\beta 1$ epitope site. The N13E mutation was the least well expressed of the mutants (Fig. 2), perhaps reflecting the known influence of the N-linked glycosylation sites on the interaction of the hCG $\beta$-chain with molecular chaperones such as BiP, Erp72 and Erp94 (Feng et al. 1996).

\section{Discussion}

We have identified key amino acids involved in the $\beta 1, \beta 6$ and $\beta 7$ epitopes on the hCG $\beta$-chain. The glutamine at position 89 and the aspartic acid at position 61 contribute to the $\beta 7$ epitope, with mutation of D61 in addition to the residues K20, 
(a)

Wild-type

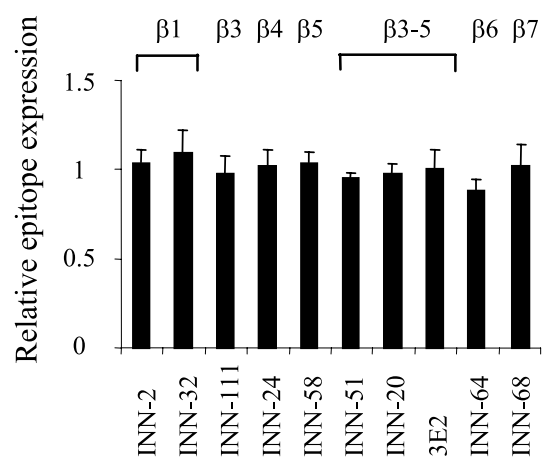

(c)

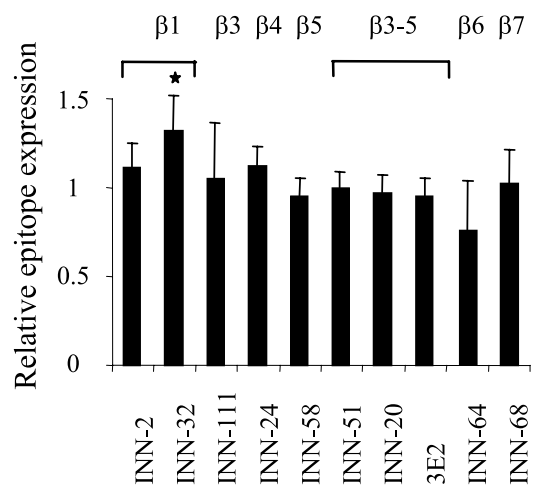

(e)

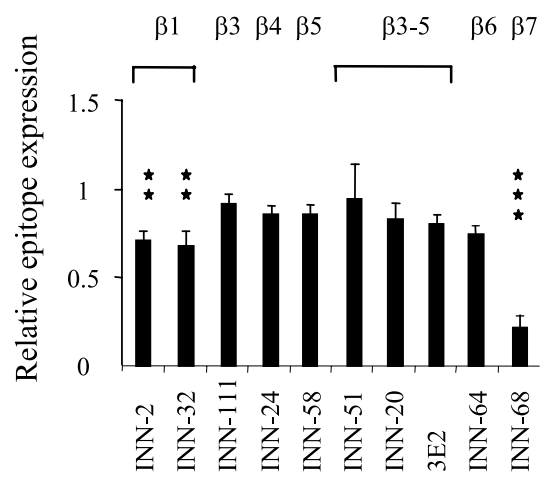

(b)

R10E

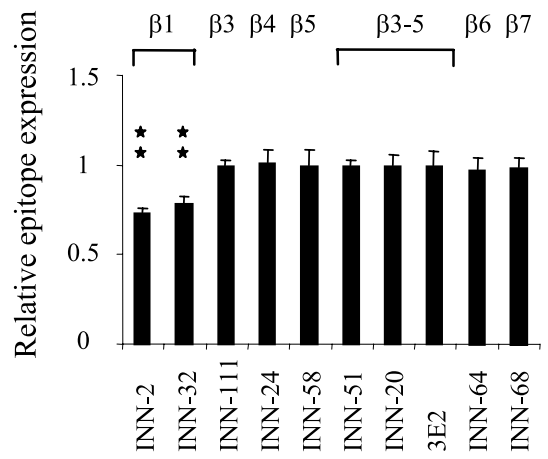

(d)

D61R

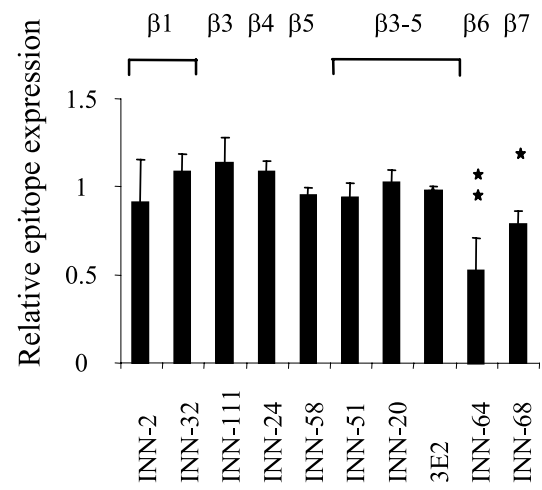

(f)

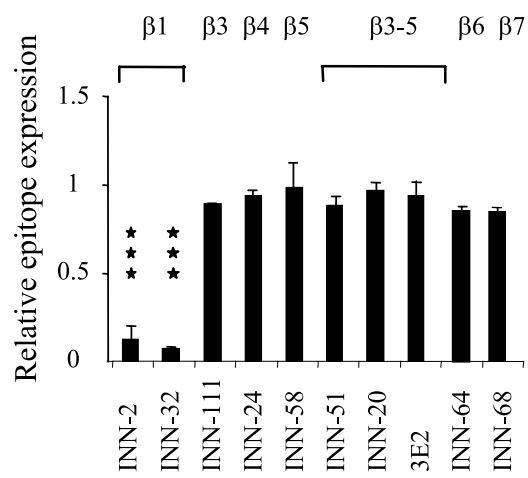

Figure 3 Epitope mapping of mutated hCG $\beta$-chains. The expression of each epitope on a mutant was assessed by the percentage of the cell population having a fluorescence intensity superior to the fluorescence intensity of the population stained with the negative isotype-matched control for each cell line stained with a panel of mAbs (INN-2

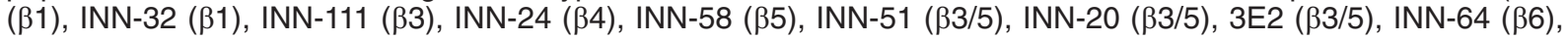
INN-68 ( $\beta 7)$ and OT3A2 (CTP)). Each flow cytometry experiment was carried out at least four times on cells optimally expressing the transfectant. To allow for variable surface expression of each transfected cell line, the relative expression of each epitope was calculated for each antibody by reference to staining of the CTP $\beta 8$ epitope, as described in Materials and methods. ${ }^{*} P<0.05$, ${ }^{* *} P<0.005$, ${ }^{* * *} P<0.0005$, comparing antibody binding to the mutant with that to the wild-type hCG $\beta$-chain. 


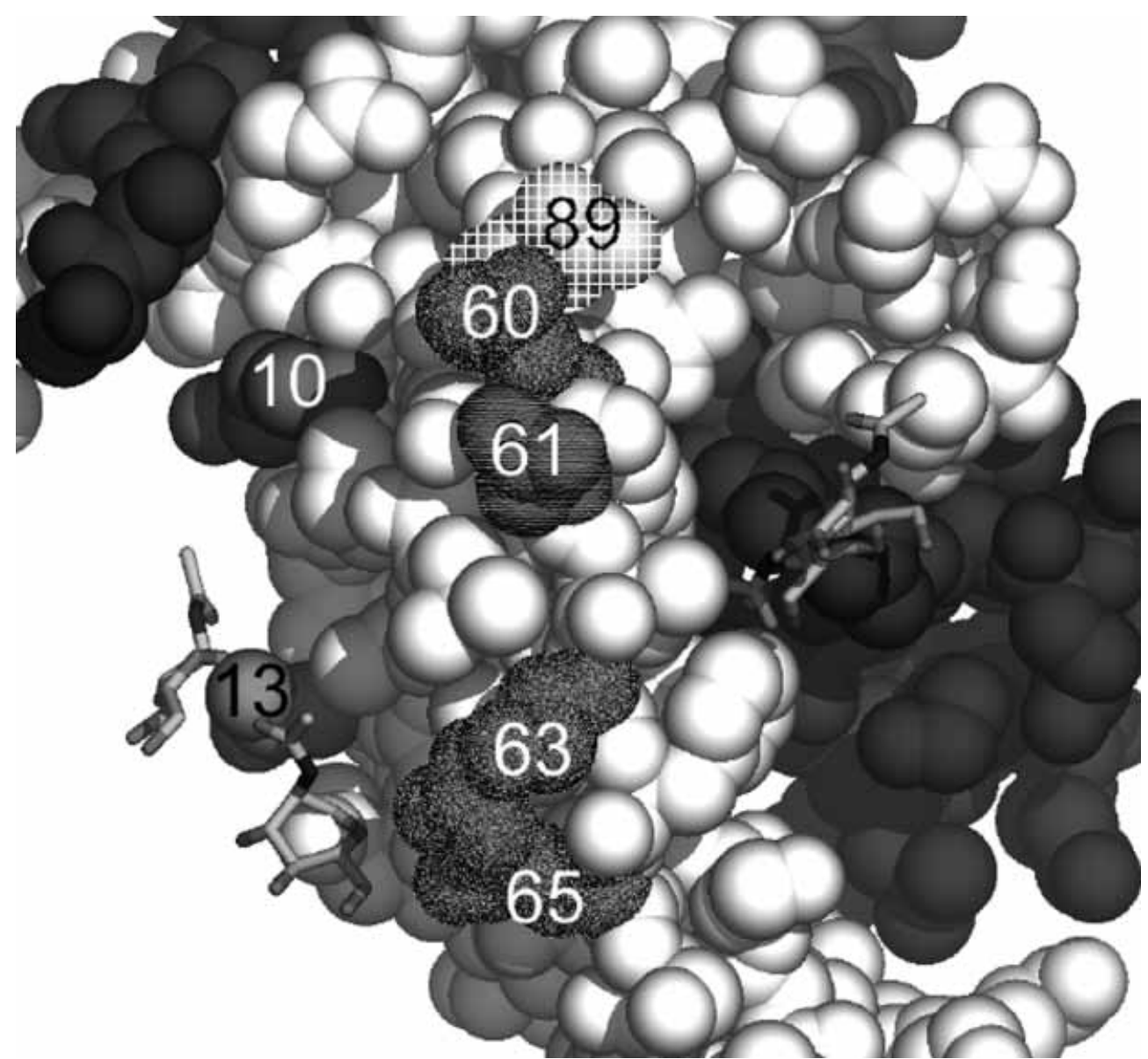

Figure 4 Location of site-directed mutations leading to decreased antibody binding. A detail of the middle of the hCG heterodimer structure represented in space-filling with the position of individual mutations highlighted. The $\alpha$-chain is shaded in dark grey and the $\beta$-chain in light grey. The $\mathrm{N}$-acetylglucosamine sugars visible in the hCG crystal structure, which are attached at N13 and N30 of the $\beta$-chain and N52 of the $\alpha$-chain, are shown in stick representation.

E21, G22 and G75 (Jackson et al. 1996) also affecting the $\beta 6$ epitope. These two epitopes are located at the $\alpha / \beta$ interface and therefore accessible only on the free $\beta$-chain.

The $\beta 1$ region represents the major hormonespecific conformation-dependent epitope on hCG (Berger et al. 2002). However, the precise location of this epitope region on the hGG $\beta$-chain has been unclear. Easily accessible areas of a protein surface, as identified by a high protrusion index and solvent accessibility, are often the site of antibody recognition (Novotny et al. 1986, Thornton et al. 1986). Analysis of the crystal structure of hCG (Lapthorn et al. 1994, Wu et al. 1994) revealed a protruding area not previously identified as an antigenic area (Berger et al. 1996). Hypothesising that this might constitute the $\beta 1$ epitope, we mutated amino acid residues at this location.

The $\mathrm{R} 60 \mathrm{H}+\mathrm{R} 63 \mathrm{~S}+\mathrm{E} 65 \mathrm{~A}$ triple mutation spans approximately $15 \AA$ of the length of the $\beta$-subunit and therefore disrupts a significant portion of the $\beta$-subunit surface that has not been investigated in previous studies (Fig. 4) (Jackson et al. 1996). This mutant totally abolishes binding of both the anti- $\beta 1$ antibodies but is in fact further down the molecule than the hormone-specific changes between hCG and LH. The R63S+E65A double mutation bound all antibodies equally as well as the wild-type hCG $\beta$-chain, clearly suggesting that the $\mathrm{R} 60 \mathrm{H}$ is the key mutation that abolishes binding of both the anti- $\beta 1$ 
antibodies. This substitution, which modifies the size but maintains the hydrophilicity and the charge, did not alter the binding of antibodies directed to any other epitopes on hGG. This indicates that there was no substantial effect on the overall conformation of the protein but that R60 establishes contact with one or more residues in the paratope of the anti- $\beta 1$ antibodies. When either the arginine residue at position 10 was changed to a glutamic acid or the glutamine at position 89 changed to an arginine, the binding of both $\beta 1$-specific antibodies was reduced to approximately $70 \%$ of the binding to the wild-type protein. Residues R60 and Q89 are adjacent in the three-dimensional structure of the hCG $\beta$-subunit and therefore would be expected to contribute to the $\beta 1$ epitope. The side-chain of R10 adopts a conformation which places it further away from the other residues implicated in the $\beta 1$ epitope. When this residue is mutated to glutamate, the side-chain is likely to adopt an orientation facing towards R60, possibly forming an electrostatic interaction. In the native heterodimer, accessibility of the residue at position 10 might be restricted by the position of the N-terminal region of the $\alpha$-chain and by the presence of the N-linked carbohydrate at position 13 on the $\beta$-chain (Fig. 4). Therefore, it is possible that the residue $\mathrm{R} 10$ does not directly bind the hypervariable regions of the INN-2 and INN-32 $\mathrm{mAbs}$ but rather is located on the periphery of the $\beta 1$ epitope and establishes positive interactions with framework residues, thereby stabilising antibody binding. Alternatively, its substitution could modify the conformation of the epitope resulting in decreased recognition. However, changing the adjacent arginine at residue 8 to a leucine did not affect the binding of the $\beta 1$-specific mAbs.

Mutation of the asparagine at position 13, one of the two $\mathrm{N}$-linked glycosylation sites of the $\beta$-chain, removes this large oligosaccharide. The loss of this carbohydrate structure was associated with increased recognition of one of the antibodies specific for the $\beta 1$ epitope, INN-32. This is most likely due to the removal of some steric hindrance of the antibody by the glycosylation.

Substitution of the aspartic acid residue at position 61 in the hCG $\beta$-chain by an arginine dramatically changes both the size and charge of the amino acid residue. However, despite its proximity to residue 60 (Fig. 4) and the fact that the $\mathrm{R} 60 \mathrm{H}$ mutation abolished binding of the anti- $\beta 1$ antibodies, the substitution D61R had no significant effect on the binding by the $\beta 1$-specific mAbs. This suggests that the carboxylate of the aspartic acid is not involved in the establishment of side-chain electrostatic interactions with the antibodies. Moreover, if the residue D61 is indeed present at the interface with the anti- $\beta 1$ antibody, the finding that both antibodies accommodated the variation of volume introduced by the D61R replacement suggests that the interface between the antibodies and hCG $\beta$-chain are not highly complementary in shape at this position. Surprisingly the substitution D61R did decrease recognition of the $\beta 6$ and $\beta 7$ epitopes. Previous work by our groups has established that residues at positions 20,21, 22 and 75 and 77 contribute to the $\beta 6$ epitope (Jackson et al. 1996, Berger et al. 2002). These residues are located in an area over $25 \AA$ from position 61 in the tertiary structure of hCG. Thus, it is unclear why the mutation of this residue modifies the recognition of the $\beta 6$ epitope. This mutation could induce an alteration of the conformation to which the $\beta 6$ epitope is sensitive or produce functional variation by indirect or long-range effects (Lavoie et al. 1992), for example by influencing the side-chain positions on loops 1 and 3 in relation to each other. However, given the absence of any effect on the other $\beta$ epitopes that are close to the $\beta 6$ epitope argues against this as an explanation. Whether the conformation of the C-terminus of the $\beta$-chain (residues 109-145) is affected by this mutation and blocks the $\beta 6$ epitope is also a possible explanation for this effect.

Insight into the position of the $\beta 7$ epitope is provided by the Q89R mutation, which dramatically reduces the binding of the INN-68 mAb. This clearly shows that Q89 is involved in antibody binding to the $\beta 7$ epitope. However this should not be over interpreted, as the introduction of arginine at residue 89 , due to its size, would be expected to have an effect over a larger region than a smaller substitution. The fact that the triple mutant including $\mathrm{R} 60 \mathrm{H}$ does not affect INN-68 binding suggests that Q89 is not the centre of the $\beta 7$ epitope. However, our finding that residue 89 plays a role in the binding of both $\beta 1$ and $\beta 7$ antibodies would accord with the previously observed close relationship between these two epitopes (Berger et al. 1990, 2002).

In discontinuous epitopes, usually 15-20 residues establish contact with the residues of the paratope 
(Jones \& Thornton 1996). A majority of these residues may be energetically significant in binding the ligand (Dall'Acqua et al. 1996, Goldman et al. 1997), or sometimes only two or three of the contact residues provide most of the binding energy (Novotny et al. 1989, Cunningham \& Wells 1993, Kelley \& O'Connell 1993). The fact that the substitution $\mathrm{R} 60 \mathrm{H}$ abolished the binding of the anti- $\beta 1$ antibody suggests that this epitope is energetically dependent on only a few residues. Moreover, this would explain the lack of effect of the substitutions of residues in close proximity on the crystal structure that might be in contact, such as the aspartic acid at position 61. Mutation of a contacting residue which does not play a significant energetic role may be accompanied by reorganisation of water molecules, which restores the original interaction (Dall'Acqua et al. 1998).

In conclusion, our results suggest that the recognition of the major hormone-specific discontinuous epitope, $\beta 1$, on hCG is based on only a very few critical interactions. We have identified these energetically dominant residues as including the arginines at positions 10 and 60, and the glutamine at position 89. Moreover, we have showed that this epitope is dramatically tolerant to substitutions of other residues probably located at the binding site, such as the aspartic acid residue at position 61 . Thus, to supplement the inherently weak immunogenicity of the hormone-unique linear CTP structures, this epitope region should be maintained in mutated or subunit vaccines aimed at optimising a hormone-specific response against hCG for control of fertility or cancer. The same considerations hold true for residues contributing to the $\beta 6$ and $\beta 7$ epitopes for vaccines which target cancers where isolated $\beta$-chains function as growth factors (Butler et al. 2000).

\section{Acknowledgements}

OT3A2 was a gift from Dr Wim Stevens (Organon Technika, Boxtel, The Netherlands) and recognises the linear CTP epitope 133-139. CHO cells were kindly provided by Dr Parmit Jat (Ludwig Institute for Cancer Research, London, UK) and cationic liposomes by Dr Karl Murray (Department of Psychiatry and Behavioural Sciences, University College London, London, UK).

\section{References}

Berger P, Klieber R, Panmoung W, Madersbacher S, Wolf H \& Wick G 1990 Monoclonal antibodies against the free subunits of human chorionic gonadotrophin. Fournal of Endocrinology 125 301-309.

Berger P, Bidart JM, Delves PJ, Dirnhofer S, Hoermann R, Isaacs N, Jackson A, Klonisch T, Lapthorn A, Lund T et al. 1996 Immunochemical mapping of gonadotropins. Molecular and Cellular Endocrinology 125 33-43.

Berger P, Sturgeon C, Bidart JM, Paus E, Gerth R, Niang M, Bristow A, Birken S \& Stenman UH 2002 The ISOBM TD-7 Workshop on hCG and related molecules. Towards user-oriented standardization of pregnancy and tumor diagnosis: assignment of epitopes to the three-dimensional structure of diagnostically and commercially relevant monoclonal antibodies directed against human chorionic gonadotropin and derivatives. Tumour Biology 23 $1-38$.

Butler SA, Ikram MS, Mathiew S \& Iles RK 2000 The increase in bladder carcinoma cell population induced by the free beta subunit of human chorionic gonadotrophin is the result of an anti-apoptosis effect and not cell proliferation. British Fournal of Cancer 82 1553-1556.

Cunningham BC \& Wells JA 1993 Comparison of a structural and a functional epitope. Fournal of Molecular Biology 234 554-563.

Dall'Acqua W, Goldman ER, Eisenstein E \& Mariuzza RA 1996 A mutational analysis of the binding of two different proteins to the same antibody. Biochemistry 35 9667-9676.

Dall'Acqua W, Goldman ER, Lin W, Teng C, Tsuchiya D, Li H, Ysern X, Braden BC, Li Y, Smith-Gill SJ et al. 1998 A mutational analysis of binding interactions in an antigen-antibody protein-protein complex. Biochemistry 37 7981-7991.

Delves PJ, Lund T \& Roitt IM 2002 Antifertility vaccines. Trends in Immunology 23 213-219.

Dirnhofer S, Wick G \& Berger P 1994 The suitability of human chorionic gonadotropin (hCG)-based birth control vaccines. Immunology Today 15 455-501.

Feng W, Bedows E, Norton SE \& Ruddon RW 1996 Novel covalent chaperone complexes associated with human chorionic gonadotropin beta subunit folding intermediates. Fournal of Biological Chemistry 271 18543-18548.

Fox H \& Kharkongor FN 1970 Immunofluorescent localisation of chorionic gonadotrophin in the placenta and in tissue cultures of human trophoblast. Fournal of Pathology 101 277-282.

Goldman ER, Dall'Acqua W, Braden B \& Mariuzza RA 1997 Analysis of binding interactions in an idiotope-antiidiotope protein-protein complex by double mutant cycles. Biochemistry 36 $49-56$.

Horton RM \& Pease LR 1991 Recombination and mutagenesis of DNA sequences using PCR. In Directed Mutagenesis, p 217-247. Ed. MJ McPherson. Oxford: IRL Press.

Jackson AM, Klonisch T, Lapthorn AJ, Berger P, Isaacs NW, Delves PJ, Lund T \& Roitt IM 1996 Identification and selective destruction of shared epitopes in human chorionic gonadotropin beta subunit. Fournal of Reproductive Immunology 31 21-36.

Jones S \& Thornton JM 1996 Principles of protein-protein interactions. PNAS 93 13-20.

Kelley RF \& O'Connell MP 1993 Thermodynamic analysis of an antibody functional epitope. Biochemistry 32 6828-6835.

Kessler MJ, Reddy MS, Shah RH \& Bahl OP 1979 Structures of $\mathrm{N}$-glycosidic carbohydrate units of human chorionic gonadotropin. Fournal of Biological Chemistry 254 7901-7908.

Lapthorn AJ, Harris DC, Littlejohn A, Lustbader JW, Canfield RE, Machin KJ, Morgan FJ \& Isaacs NW 1994 Crystal structure of human chorionic gonadotropin. Nature 369 455-461.

Lavoie TB, Drohan WN \& Smith-Gill SJ 1992 Experimental analysis by site-directed mutagenesis of somatic mutation effects on affinity 
and fine specificity in antibodies specific for lysozyme. Fournal of Immunology 148 503-513.

McQuillin A, Murray KD, Etheridge CJ, Stewart L, Cooper RG, Brett PM, Miller AD \& Gurling HM 1997 Optimization of liposome mediated transfection of a neuronal cell line. Neuroreport $\mathbf{8}$ 1481-1484.

Moulton HM, Yoshihara PH, Mason DH, Iversen PL \& Triozzi PL 2002 Active specific immunotherapy with a beta-human chorionic gonadotropin peptide vaccine in patients with metastatic colorectal cancer: antibody response is associated with improved survival. Clinical Cancer Research 8 2044-2051.

Novotny J, Handschumacher M, Haber E, Bruccoleri RE, Carlson WB, Fanning DW, Smith JA \& Rose GD 1986 Antigenic determinants in proteins coincide with surface regions accessible to large probes (antibody domains). PNAS 83 226-230.

Novotny J, Bruccoleri RE \& Saul FA 1989 On the attribution of binding energy in antigen-antibody complexes McPC 603, D1·3, and HyHEL-5. Biochemistry 28 4735-4749.

Seed B 1987 An LFA-3 cDNA encodes a phospholipid-linked membrane protein homologous to its receptor CD2. Nature 329 840-842.

Sheppard AJ, Cussell D \& Hughes M 1984 Production and characterization of monoclonal antibodies to tetanus toxoid. Infection and Immunity 43 710-714.
Stevens VC 1999 Antifertility vaccines. In Handbook of Experimental Pharmacology 133, pp 443-461. Eds P Perlmann \& H Wigzell. Heidelberg: Springer-Verlag.

Talwar GP 1999 Vaccines and passive immunological approaches for the control of fertility and hormone-dependent cancers. Immunological Reviews 171 173-192.

Talwar GP, Singh O, Pal R, Chatterjee N, Sahai P, Dhall K, Kaur J, Das SK, Suri S \& Buckshee K 1994 A vaccine that prevents pregnancy in women. PNAS $918532-8536$.

Thornton JM, Edwards MS, Taylor WR \& Barlow DJ 1986 Location of 'continuous' antigenic determinants in the protruding regions of proteins. EMBO Fournal 5 409-413.

Triozzi PL \& Stevens VG 1999 Human chorionic gonadotropin as a target for cancer vaccines. Oncology Reports 6 7-17.

Wu H, Lustbader JW, Liu Y, Canfield RE \& Hendrickson WA 1994 Structure of human chorionic gonadotropin at $2 \cdot 6 \AA$ resolution from MAD analysis of the selenomethionyl protein. Structure $\mathbf{2}$ $545-558$.

Received 15 October 2003

Accepted 8 December 2003 